\title{
Tangence
}

\section{Acadie City ou l'invention de la ville}

\section{François Paré}

Numéro 58, octobre 1998

Le postmoderne acadien

URI : https://id.erudit.org/iderudit/025977ar

DOI : https://doi.org/10.7202/025977ar

Aller au sommaire du numéro

Éditeur(s)

Tangence

ISSN

0226-9554 (imprimé)

1710-0305 (numérique)

Découvrir la revue

Citer cet article

Paré, F. (1998). Acadie City ou l'invention de la ville. Tangence, (58), 19-34.

https://doi.org/10.7202/025977ar d'utilisation que vous pouvez consulter en ligne.

https://apropos.erudit.org/fr/usagers/politique-dutilisation/ 


\section{Acadie City ou l'invention de la ville François Paré}

Que l'Acadie ait produit, depuis le milieu de ce siècle, une culture de l'imaginaire, voilà qui me semble motiver une grande part de la poésie acadienne des vingt-cinq dernières années. Depuis Cri de terre (1972) de Raymond Guy LeBlanc jusqu'à Nocturnes (1997) de Serge Patrice Thibodeau, cette poésie très abondante, très présente dans l'ensemble du discours culturel acadien, exprime à la fois le triomphe de l'imaginaire sur le réel, seule condition de l'affirmation identitaire, et la lutte persistante contre la menace de l'irréalité qui condamne peut-être — on n'en sait rien, au fond - toute la collectivité à se dissoudre dans sa parole même. Et si l'Acadie n'existait pas? En dépit de tout ce qui est dit et proclamé à son sujet et en son nom? Le doute est omniprésent. Et la parole, qu'on voulait si prégnante du réel, verse dans la pure représentation:

Grillages rouillés pour unique mémoire,

Nous mentons. Nous mentons, nous renions

Les mots qui nourrissent : emmanchure, malpatient,

Coulée. Passons la porte,

Prenons notre erre pour aller nulle part. ${ }^{1}$

Mensonge et errance! Prendre son erre! Voilà ce qui semble ici nourrir le sujet acadien: c'est en tout cas la logique implacable de son irréalité et de son déplacement, comme si d'étranges forces de dispersion - suradéquation et inadéquation devant le réel -, si présentes dans l'histoire du peuple acadien, continuaient d'habiter au coeur même du discours poétique pour en constituer les formes les plus pressantes et surtout les plus résolument excessives.

Sauvegarde ambiguë, empoisonnée donc, que la littérature! "Il y a des légendes, écrit Herménégilde Chiasson, qui deviennent si prenantes qu'on oublie d'en préciser la fiction, d'en départager le folklore du réel, comme si l'on craignait que tout ne bascule soudain dans la perte et la démence de renaître à l'inouï" ${ }^{2}$ En

1 Serge Patrice Thibodeau, Noctumes, Trois-Rivières, Écrits des Forges, 1997, p. 41.

2 Herménégilde Chiasson, Vous, Moncton, Éditions d’Acadie, 1991, p. 105. 
1972, en pleine effervescence culturelle à Moncton, Alain Masson s'élevait contre la vision figée de l'Acadie qu'une certaine critique québécoise (représentée par Georges-André Vachon et Jean-Guy Pilon) véhiculait alors. Masson imagine une autre Acadie: "Lorsque les Acadiens écrivent, ils donnent à leur pays une matière de langue qui devrait triompher du mythe, en lui opposant utilement une légende: une version lisible d'eux-mêmes. . 3

Arrivé à Moncton en 1968, Masson découvre à travers le questionnement et les luttes quotidiennes qui marquent alors la vie acadienne au Nouveau-Brunswick une communauté lui rappelant ses racines bretonnes. Sa lecture d'une nouvelle poésie acadienne, certes influencée par sa propre ambivalence envers sa culture d'origine, lui permet de comprendre la complexité du mouvement d'affirmation en Acadie et surtout le rôle majeur qu'y joue la poésie. Or, dans tout cela, Masson saisit admirablement bien l'urbanité de la nouvelle écriture. Car, à ses yeux, l'Acadie doit éviter à tout prix la folklorisation qui a été le fait de la renaissance bretonne. Ce qui fait tout l'intérêt du réveil minoritaire au Nouveau-Brunswick, c'est qu'il est, à l'inverse, croit-il, un mouvement issu de la ville et fortement nourri par les ambiguités du milieu urbain. Masson théorise donc la naissance d'une "civitas ", à la manière de la Rome antique: une ville qui, plus que le rassemblement aléatoire de milliers d'individus, deviendrait le centre d'une citoyenneté acadienne au sens fort. "L'État est une institution", écrit-il en 1972 dans un condensé lapidaire, "la cité une incarnation de la vie commune. " ${ }^{4}$ La communauté acadienne, ne pouvant et surtout ne voulant trouver son affirmation dans les structures étriquées de l'État politique, se prend donc à reporter son désir de cohésion sur la figure emblématique de la ville, espace d'une nouvelle civilité en Amérique du Nord. "L'Acadie, poursuit Masson dans le même texte, doit devenir une cité dans l'État [...]. Il n'est pas question de définir les droits d'une pareille cité, il ne s'agit que d'y reconnaître l'expression démocratique d'un peuple dont personne n'ose aujourd'hui contester l'existence et l'originalité. Il suffit que les Acadiens se disent et se constituent Acadiens. "5 Ces textes d'une extraordinaire lucidité

3 Alain Masson, Lectures acadiennes. Articles et comptes rendus sur la littérature acadienne depuis 1972, Moncton/Luxembourg, Perce-Neige/L'Orange bleue, 1994, p. 52. Je souligne.

4 Ibid., p. 106.

5 Ibid., p. 110. 
tendent alors à concevoir autrement la quête identitaire. L'Acadie ne sera pas un État, instance purement administrative qui n'intéresse ni la littérature, ni le "peuple acadien" dans son agir quotidien. Elle ne sera pas non plus un avatar folklorique. Plutôt, "par une agglomération progressive $[\ldots]$, par une cooptation vécue dans les quartiers " 6 , la communauté acadienne prendra les contours d'une urbanité civile et civilisée, loin de l'enfermement national, loin des "gadgets vendus aux touristes " 7 . Issue d'une littérature résolument urbaine, l'Acadie sera le produit de l'action poétique, expression singulière de la conscience, nécessaire à l'émergence de la "civitas": "l'Acadie [sera] la négativité au travail " 8 . Cette "cité acadienne" divergerait largement, du reste, de l'imaginaire nationaliste au Québec, puisque celui-ci, se détachant plutôt idéologiquement de Montréal à la fin des années soixante, a mis tous ses espoirs dans la formation à plus ou moins long terme d'un État administratif national.

Ainsi se formulait alors le projet singulier de toute une génération de poètes, qui, au "mythe" enfermant d'une Acadie villageoise et taciturne, celui que véhiculait encore largement le discours culturel acadien", voudrait substituer la "légende" d'une collectivité "arrivée en ville", qui ferait un "tintamarre", et se donnerait ainsi bruyamment à lire :

Aurions-nous perdu le goût des rives vertes

De ces villes de promesses

Enfouies dans nos mémoires d'hommes. ${ }^{10}$

\section{Ibid.}

7 Ibid., p. 55.

8 Ibid., p. 56.

9 Voir, par exemple, les dernières pages du livre de Régis Brun, qui date pourtant de $1982 \times$ Pays de mer et de dunes de sable, de prés et d'aboiteaux, de caps et de havres! Pays de champs d'avoine et de patates, de grands sapins et de bouleaux blancs, de bourrasque de neige et de journées ensoleillées d'été qui n'en finissent plus.... (Régis Brun, De Grand-Pré à Kouchibougouac: l'bistoire d'un peuple exploité, Moncton, Éclitions d'Acadie, 1982, p. 172).

10 Raymond (Guy) LeBlanc, Cri de terre, Moncton, Éditions d'Acadie, [1972], 1992, p. 54. Raoul Boudreau offrait, dans un colloque récent, une analyse très fine de cette opposition entre mutisme et surabondance de la parole clans le roman récent en Acadie: - La folie, la litote, l'hyperbole: trois rapports à la langue dans le roman acadien •, Colloque * Les langues du roman: Du multilinguisme comme stratégie textuelle., Université de Montréal, 10 octobre 1997, texte inédit. Je ne conserve, quant à moi, que le doublet litote-hyperbole, car la *folie - ne me semble pas faire partie de lunivers poétique acadien actuel. Je lui suis reconnaissant de mavoir lancé sur une bonne piste. 
Acadie City, ce serait là l'espace urbain où une errance fondatrice trouverait à s'accomplir en toute fidélité avec elle-même. La ville inventée, créée de "nulle part", représenterait, pour la collectivité minorisée, la synthèse de tous les espoirs d'accéder un jour à la parole publique, c'est-à-dire de faire de la dispersion l'expression d'une vivante "lisibilité". La cité acadienne, ce serait justement cette "Acagonie" des recommencements, dont rêvait, en 1976, Herménégilde Chiasson dans le Rapport sur l'état de mes illusions.

Plus vulnérable aux vicissitudes de la mémoire, à la perte qui lui semblait au coeur de toute histoire individuelle et collective, la poésie acadienne a donc cherché à reconstituer autrement, dans la fluidité, l'espace identitaire. Et c'est dans un ailleurs urbain, une métropole de l'imaginaire, productrice d'altérité, qu'elle a été amenée à comprendre le déracinement identitaire qui, seul, semblait faire d'elle une culture du temps présent. Il y avait là, certes, de grands dangers de déperdition culturelle: d'où le cri de Raymond Guy LeBlanc dans son évocation de Petitcodiac: "Tout un peuple se désacadise au béton Albion. " ${ }^{11}$ Mais il fallait justement peut-être que l'Acadie se "désacadise" pour enfin glisser dans la légende, dans le lisible. The legend lives on, lisait-on alors dans les réclames américaines des années soixante-dix: "L'errance est un continuel présent "12, annonçait à son tour le poète, devenu habitant quintessentiel de la "cité". Ainsi la "grande cage à nostalgie " ${ }^{13}$ d'Eugénie Melanson appartenait désormais au passé. Et, par une curieuse transmutation, Moncton deviendrait, dans la rupture avec le mythe, une "cité / city " non seulement métonymique, éclatée, fragmentée, mais aussi accueillante de la marginalité de ses chantres, une hypostase de la république des lettres, aussi légendaire, il va sans dire, que le pays lui-même.

Ce qui m'intéresse dans la lecture de certains textes marquants de la littérature acadienne, depuis le milieu des années soixante-dix, c'est donc la construction progressive de la légende monctonienne. Et, au-delà de Moncton, nous le verrons, le surgissement de l'espace urbain tout entier dans la construction identitaire du sujet minoritaire. Moncton, ce n'est pas l'Acadie, pas plus que Montréal n'est le Québec, comme on voudra vite me le rap-

11 Ibid., p. 59.

12 Gêrald Leblanc, Les matins habitables, Moncton, Perce-Neige, 1991, p. 23.

13 Herménégilde Chiasson, Mourir à Scoudouc, Moncton, Éditions d'Acadie, 1974 , p. 35. 
peler. C'est incontestable. Mais Moncton s'est constituée tout de même au cours des vingt-cinq dernières années comme un espace de référence prioritaire qui, pour le lecteur que je suis, doit faire l'objet d'un questionnement ${ }^{14}$.

Quelle est donc la nature de cette "cité"? C'est une double nature. En 1970, le déplacement de l'espace identitaire vers la ville est motivé pour les uns par une exigence de liberté individuelle et collective. C'est ainsi que Gérald Leblanc interprète la quête de quotidienneté dans les premiers textes monctoniens de Raymond Guy LeBlanc: par exemple, La densité du réel et Cri de terre de ce "poète fondateur" lui paraissent être des œuvres de résistance: " une tentative de réapprendre à respirer, à évacuer les restants de peur et d'angoisse existentielle, bref à définir un espace sensible où continuer d'évoluer" "'. C'est ainsi que l'on voudra bien lire, jusqu'à aujourd'hui, Acadie Rock de Guy Arsenault. Moncton serait donc devenue, dans ces textes, le lieu de la pleine prise en charge de l'individualité et de son inscription dans un cadre civique qui en assurerait la fluidité et la libre affirmation.

Mais l'espace urbain est rempli à satiété de violence. Il est non seulement liberté et euphorie, mais souillure et luttes fratricides. Comment donc y chercher refuge, y loger les conditions de l'identité? Nulle allégresse chez un poète comme Herménégilde Chiasson: ici l'urbanité acadienne, bien que pressante, évoquera plutôt la douloureuse impuissance à vivre dans le réel. Tout ne se passe-t-il pas, au contraire, dans le langage? "Il y avait cette ville si grande que personne n'aurait rêvé y faire le voyage et il y avait cette ville si terrible et angoissante que le cour en restait saisi quand on y mettait les pieds." Aussi la ville "légendaire", où se produit dans un certain

14 Cet espace de référence s'inscrivait dans un long processus d'appropriation de Moncton par la migration acadienne, un processus complexe, parfois très tourmenté, dont Phyllis E. Leblanc (*Idéologie nationale et intégration des francophones dans un contexte urbain: le cas de Moncton "Daniel Hickey (dir.). Moncton 1871-1929. Changements socio-économiques dans une ville ferroviaire, Moncton, Éditions d'Acadie, 1990, p. 129-161) rend bien compte. Ce qui est intéressant dans cette étude, c'est le portrait d'une population acadienne rapidement urbanisée à Moncton, - en dépit des barrières culturelles ou idéologiques et même, à l'occasion, avec l'appui concret des élites francophones urbaines de Moncton* (p. 132). En ce sens, l'appropriation poétique de Moncton refléterait un mouvement plus général.

15 Gérald Leblanc, *Pour Raymond Guy LeBlanc*, Raymond Guy LeBlanc, La mer en feu, Moncton, Perce-Neige, 1993, p. 8.

16 Herménégilde Chiasson, Vous, op. cil., p. 137. 
24

flamboiement de l'individualité et dans la perte du nous collectif la renaissance identitaire n'en reste-t-elle pas moins, pour Chiasson, le lieu d'une profonde inadéquation aux choses et aux êtres.

C'est que la ville est un magma de contradictions. C'est en cela qu'elle séduit profondément et qu'elle s'offre suprêmement à la poésie en tant qu'espace de rédemption pour tous les opprimés. Dans le cas d'Arsenault et de Chiasson, la conjoncture de l'espace est donc la même: l'œuvre poétique repose sur une hypertrophie de la ville, de la "cité / city" nord-américaine, représentée par Moncton, et c'est derrière les appels d'une telle invention du lieu urbain que continuent de circuler, chez Gérald Leblanc, chez Herménégilde Chiasson, chez Dyane Léger, chez Ulysse Landry, chez Daniel Dugas, chez Serge Patrice Thibodeau, entre autres, de puissantes évocations de l'espace identitaire. C'est Moncton qui se dessine d'abord (surtout chez Gérald Leblanc), puis nous glissons vers l'ailleurs: New York City, Harlem, la Nouvelle-Orléans / New Orleans, San Francisco, New Delhi, Jérusalem (ancienne et nouvelle). "Les noms de villes sont des mantras", écrit Gérald Leblanc dans Les matins babitables ${ }^{17}$. Face au village en déclin, il y a dans la litanie des villes un capital symbolique essentiel.

C'est d'abord dans toute l'ouvre poétique de Gérald Leblanc, du reste, que se remarque avec la plus grande insistance cette construction de la ville. En fait, depuis les premiers textes, Moncton ne cesse d'occuper une place de plus en plus grande dans cette cuvre, comme si, condition même de l'expression poétique, elle était devenue pour Leblanc le seul sujet véritable de la poésie. Ici l'existence urbaine séduit par l'absence de frontières linguistiques, par le chatoiement des identités, par sa subversion apparente de toutes les normes sociales, par l'omniprésence du bruit et de la musique. D'un côté, la ville de la marginalité est un produit du rêve, une projection du désir: nombreuses sont les références, dans les poèmes de Complaintes du continent ou encore dans Éloge du chiac, à la métaphore absolue du désir que constitue l'espace urbain. On en retrouve également l'écho dans de nombreux textes plus autobiographiques, comme, par exemple, la préface écrite pour l'Événement Rimbaud. Là où un certain imaginaire enfilait volontiers le collier des noms de villages acadiens, forgeant ainsi un territoire identitaire familier, le discours 
du poète, lui, excède nécessairement les lieux restreints de la naissance. C'est ainsi, dans la rêverie urbaine, que s'est formée une communauté en poésie: "on passait l'après-midi, les orteils dans le sable, à énumérer toutes les villes qui nous fascinaient: San Francisco, Barcelone, New Orleans, Tokyo" (p. 10). D'un côté, la ville inventée au rythme des errances quotidiennes, c'est Moncton, celle des amis, rêvant de départ au Cap-Pelé, des confrères en poésie, des patronymes secrets cachés derrière l'image de chaque porte familière; de l'autre, ce sont toutes les villes qui, par définition et par extension, accueillent le poète errant dans la dérive incessante de son travail sur le sens.

Les choses ne sont donc pas si simples. En effet, si la ville reflète l'éclatement des gestes figés et annonce la liberté tant souhaitée ("la rue comme une méditation sur le mouvement "18), elle est aussi, pourtant, l'écho de solidarités anciennes qui continuent de converger en elle. Dans bon nombre de textes de Gérald Leblanc, le subterfuge est tel que l'urbanité radicale ne donne pas naissance, comme on aurait pu le penser, à un je résolument individualiste, affirmant à qui voudrait l'entendre sa singularité princière, son rejet lucide de la communauté; la ville continue de protéger certains élans de la mémoire, qui remontent en elle à intervalles réguliers. La rivière Petitcodiac, entachée de nostalgie, résume bien ce qui fait de Moncton un espace privilégié pour Leblanc: un espace où, gestionnaire de la nostalgie, le sujet urbain peut désormais s'accomplir au sein de nouvelles communautés identitaires. Dans toute l'œuvre de Gérald Leblanc, l'espace urbain renforce ainsi, contre toute attente, le nous identitaire, un nous, non plus muet et stérile, mais bavard et excessif:

les musiques rouges que tu proposes

c'est bien la lumière émanant de nous

une cadence au coeur comblé

dans l'écho neurologique de la ville

à corps perdu dans le vertige

c'est la nuit dans notre ville sauvage

dont nous chantons les excès et les chutes

où nous apprenons à dire nous

parmi les débris du monde ${ }^{19}$

18 Gérald Leblanc, Éloge du chiac, Moncton, Perce-Neige, 1995, p. 93.

19 Gerald Leblanc, Complainte du continent. Poèmes 1988-1992, Moncton/ Trois-Rivières, Perce-Neige/Écrits des Forges, 1993, p. 23. 
Ce sont ici des solidarités restreintes: celles du groupe d'amis, les chums en poésie, les errants. Est-ce bien cela l'Acadie "arrivée en ville"? Le poète se contente de maintenir l'ambiguité. Sauvage et violente, la ville invente ou même protège les formes pérennes de la communauté. C'est au bord de la perte que nous nous reconnaissons, au moment où, fracturée, la communauté opprimante de la naissance s'ouvre sur l'imaginaire. Lieu du choix lucide de l'identité, la ville n'empêche pas la nostalgie, chacun continuant de songer aux anciennes allégeances, si terriblement univoques. En même temps, l'œuvre de Gérald Leblanc ne cesse jamais d'offrir l'image d'une urbanité ouverte, celle que décrit et souhaite à son tour Joseph Yvon Thériault dans sa rêverie sur l'avènement d'une Acadie postmoderne: il y aurait alors "une identité francophone dorénavant moins globalisante, plus malléable, moins fondée sur la pesanteur de l'histoire et plus sur la légèreté de l'être dans son rapport quotidien avec l'autre" 20 . Ces textes sont éclairants. Car l'histoire est lourde pour Thériault: l'Acadie n'a d'histoire que nostalgique où, chant, complainte, silence, elle n'est pas lisible dans les termes propres à la modernité. Il faut donc accéder à une Acadie allégée des "lourdeurs" de son histoire, prête à jouer le jeu quotidien de sa disparition, dans le théâtre d'une lutte "légère " et fraternelle avec l'Autre.

Chez Herménégilde Chiasson, les choses ne se présentent pas tout à fait de la même manière. La ville est chez cet écrivain l'objet d'une méfiance paradoxale. D'une part, elle se construit explicitement comme un lieu d'oppression linguistique, une entrave à la cohérence de la parole poétique. En effet, là où nombre de textes de Gérald Leblanc annoncent l'avènement de la "ville-langues / Moncton on my mind" 21 , les écrits de Chiasson, tout comme ceux d'Ulysse Landry, du reste, sont passablement plus nuancés et plus pessimistes. C'est qu'une grande part de l'œuvre de Chiasson constitue une condamnation sans équivoque du "mythe" de l'Acadie fière et survivante. La ville bilingue — et non pas créole, comme chez Léger et Leblanc, par exemple est, pour lui, un lieu de déperdition culturelle. D'autre part, seule

20 Joseph Yvon Thériault, L'identité à l'épreuve de la modemité. Écrits politiques sur l'Acadie et les francophonies canadiennes minoritaires, Moncton, Éditions d'Acadie, 1995 , p. 88.

21 Gérald Leblanc, Complaintes du continent, op. cit, p. 24. 
Moncton, en dépit de sa "cartographie du vide" 22 , peut pourtant entrouvrir l'espace restreint d'une Acadie traditionnelle et enfermante. Moncton est pour Chiasson un mal nécessaire. C'est que la ville anglaise engendre une conscience aiguë de l'identité (perdue?) et, par delà, une quête existentielle de l'Autre.

se réveiller à cette réalité déconcertante

devant vous à quelques mètres à peine

un homme vient d'éclater de rire

seul dans le métro

son cơur vient lui aussi d'éclater

une déflagration

dans les cercles concentriques de sa conscience ${ }^{23}$

On le voit donc: l'espace urbain fracture la belle unité antérieure et c'est dans cet éclatement que la conscience, paradoxalement, se concentre sur elle-même. Sur fond nécessaire de la nostalgie (le célèbre "tableau de backyard" de Guy Arsenault), voilà que se produit le mouvement vers l'avant, la gestion d'une absence.

C'est donc dire que Moncton est, dans toute l'ouvre de Chiasson, un espace à la fois d'extrême menace et d'extrême rédemption: le poète aime se représenter l'approche de la ville, sur l'autoroute, alors qu'il quitte le leurre du village. Au volant de sa voiture, la ville est alors pure conscience, pure "acuité " 24 . Et puis on finit toujours par pénétrer dans l'antre urbain et s'y perdre dans la confusion qui règne absolument chez tous ses habitants: "pourquoi alors s'appliquer à se rapprocher / pourquoi se laisser absorber si l'on a perdu en soi / le sens de toute direction absolue "25. Dans des textes plus récents, c'est sur cette nostalgie reniée, puis l'"absence" que Chiasson s'interroge encore: "La réalité ne pouvait se mesurer à la nostalgie. Nous savons tous que la mémoire modifie les bavures dans les virages. Contraint l'espace.

22 Herménégilde Chiasson, Prophéties, Moncton, Michel Henry éditeur, 1986 , p. 46.

23 Herménégilde Chiasson, Vous, op. cil., 1991, p. 13.

24 On retrouve un même sentiment de perte et une même recherche de l'acuité : chez Michel Roy: "Nous nous leurrons sans fin, écrit-il dans L'Acadie perdue, L'acuité est absente de notre vision de nous-mêmes. La conscience de l'effritement nous échappe. Ou plutôt l'effritement nous dégrade au point que nous avançons les yeux mi-clos, la conscience fermée, faisant des pas indécis, prenant les vapeurs de nos illusions pour les formes du réel* (L'Acadie perdue, Montréal, Québec/Amérique, 1978, p. 171).

25 Herménégilde Chiasson, Vous, op. cit., p. 29. 
Il est revenu hanté de sa présence. [...] Il est revenu faire l'archéologie de son absence." ${ }^{26}$ Si la ville nous appelle, ce n'est donc pas pour nous pousser en avant, toujours vers un ailleurs dépaysant, mais pour nous rappeler à l'ordre de l'"archéologie", cette "archéologie" n'étant, chez Herménégilde Chiasson, qu'une conscience exacerbée de l'identité perdue. Est-ce précisément un même exercice du "déregard" qu'Herménégilde Chiasson évoquait déjà si vivement dans le Rapport sur l'état de mes illusions? Certes, dans ce recueil, la "Lettre d'amour dans un pays incertain ", par sa terrible ambiguité à l'égard de la communauté d'origine, s'articulait sur l'acte délibéré de la conscience: "laisser son regard dévaler vers ce qui pourrait être l'ouverture " 27.

Mais l'évocation de la ville, dans tous ses états, dépasse largement, chez ces deux écrivains, les écrits poétiques eux-mêmes. Herménégilde Chiasson et Gérald Leblanc sont les auteurs d'un grand nombre de préfaces, de postfaces, d'introductions, de conférences publiques où se trouve confirmée, de manière encore plus cohérente, la représentation singulière de la ville. Ainsi, pour ne donner ici que cet exemple, Chiasson et Leblanc encadrent tous deux de leurs signatures la reprise d'Acadie Rock par les Éditions d'Acadie en 1994. Or la lecture qu'ils font du recueil de Guy Arsenault est extrêmement révélatrice. Dans sa préface, Chiasson rappelle l'étouffement linguistique et l'invisibilité qui, à Moncton, caractérisaient la communauté acadienne: "Guy Arsenault vit dans une ville anglaise où le tiers des Acadiens sont sans visage. À Moncton, il n'y a pas un seul nom de rue en français, les commerces annoncent en anglais, on prend votre commande et votre argent en anglais. ${ }^{28}$ Dans ce milieu étouffant, Chiasson se rappelle l'amitié des artistes monctoniens, le modèle des Noirs américains, la construction dans les années soixante-dix d'une communauté intellectuelle, que seule la ville - par ses contradictions - rendait possible. Rebelle, la ville se substituait à cette autre communauté à la docilité maladive.

D'un autre côté, la postface de Gérald Leblanc se présente, par son titre, sous le signe de la "dérive". Ici se concrétisent les

26 Herménégilde Chiasson, Miniatures, Moncton, Perce-Neige, 1995, p. 43.

27 Herménégilde Chiasson, Rapport sur l'état de mes illusions, Moncton, Éditions d'acadie, 1976 , p. 53.

28 Herménégilde Chiasson, "Relire Guy Arsenault ", Guy Arsenault, Acadie Rock, Moncton/Trois-Rivières, Perce-Neige/Écrits des Forges, 1994, p. 8. 
représentations d'un certain Moncton étudiant, au début des années soixante-dix: l'école primaire, la polyvalente, l'université, le "Joint", le " 64 Beechwood Terrace où vivaient à l'automne 1972 Raymond LeBlanc et Herménégilde Chiasson", l'horizon de Shédiac, vécu dans la plus grande ambivalence, et enfin les lieux sacrés de Memramcook. Or ce Moncton étudiant, c'est aussi celui des lectures des poètes afro-américains, et déjà les éléments d'une hypertrophie de la ville qui occupera le centre de toute l'œuvre de Leblanc. Ce qui étonne, pourtant, c'est que la lecture même d'Acadie Rock - du moins par le lecteur "étranger" que je suis - ne permet pas de confirmer l'image euphorique de la ville. L'univers de Guy Arsenault est résolument celui de la campagne: c'est encore, au cour de la ville, le village, avec ses rituels religieux et sa convivialité obsessive, qui est scandé dans le "tableau de backyard": non pas la ville effervescente et incertaine, comme aimeront l'imaginer Gérald Leblanc et Herménégilde Chiasson (et Daniel Dugas également, par exemple), mais la ville douloureusement jalouse de ses nostalgies inexprimées. Si Moncton donne "faim de l'Acadie", chez Arsenault, c'est que l'enracinement dans cette Acadie rurale et ritualisée n'y persiste qu'à l'état de désir, à l'état d'irréalité.

Or, c'est justement parce que Moncton ("Monckton" dans le lexique de Gérald Leblanc) n'est pas à proprement parler une ville acadienne qu'elle est à même de fracturer provisoirement le nœud des identités fermées et de s'ouvrir à l'Amérique. Chez Gérald Leblanc, par exemple, Moncton / Monckton n'est "habitable" que si la ville excède ses frontières immédiates et accepte de porter en elle les métropoles (impensables en Acadie!) de la modernité. Moncton est ainsi métonymique, seuil nécessaire vers d'autres villes qui l'irradient. Curieux emblème, donc, d'une métropole que l'Acadie n'aura jamais! Métropole du pur imaginaire urbain! Par la juxtaposition des villes, en signature spatiotemporelle du poème ("Robichaud-Moncton-New York City, juillet 1991-avril 1993", dans Éloge du chiac, par exemple), Leblanc attire l'attention du lecteur sur le caractère merveilleusement métamorphique de l'espace monctonien. Non seulement les individus s'y trouvent libérés des vieux carcans collectifs, mais c'est l'espace lui-même, si désespérant au départ, qui s'est mis à acquérir les vertus magiques de la transmutation. C'est ainsi que le poète ne signe plus de son nom propre, maintenant sans importance, mais de celui des villes où il s'est dispersé (ce que 
30

feront avec lui nombre d'autres poètes acadiens). Ici New York - toujours désignée comme "New York City" - (et à un moindre degré la Nouvelle-Orléans / New Orleans) permet toujours de signer le poème dans un ailleurs, le poète se représentant à luimême et à ses lecteurs comme un "ange en transit" 29 entre la grande Amérique et cette Amérique restreinte qu'est l'Acadie.

Il est certain que le rapport privilégié entre Moncton et New York - un rapport tout de même assez spectaculaire quand on considère le rayonnement réel de Moncton! - constitue un important motif pour Gérald Leblanc, par exemple. L'évocation de "New York City" s'inscrit, bien entendu, dans le cadre plus large d'une culture acadienne affirmant son américanité. La présence active de la métropole américaine permet de métamorphoser l'espace trop restreint de la communauté d'origine et de penser Moncton à partir d'une tout autre échelle de grandeur.

Cependant, au-delà de New York, la ville devient, chez Gérald Leblanc surtout, la métonymie de tout l'axe acadien de la dispersion. Expulsée du "mythe"fondateur, la ville inventée y revient invariablement. Et ce sera à travers toute l'Amérique que le poète recensera, une à une, à la suite de Moncton, les métropoles où se logera provisoirement son écriture, Elles sont nombreuses, ces métropoles inventées. Ce sera d'abord, chez Leblanc et chez Léger notamment, la Nouvelle-Orléans où surgit l'identification avec le Noir américain, celui du blues surtout, celui de l'affirmation contre l'oppression. La Nouvelle-Orléans est évidemment proche à tous les points de vue de l'Acadie. Il est normal que Moncton en soit le réceptacle métonymique. Mais, il y a plus, puisque le poète acadien affirme par là sa participation privilégiée à la culture afro-américaine. Héritier de W.E.B. Dubois et d'Allen Ginsberg, il croit comprendre le langage cryptique de cette culture mieux que tout autre et se sent accueilli par elle comme s'il en avait toujours fait partie.

Dyane Léger a fait des représentations de la Nouvelle-Orléans - appelée "New Orleans", comme chez Gérald Leblanc - le centre de certains écrits plus récents. Les anges en transit donne lieu, par exemple, à des glissements spectaculaires de l'identité. Possédée par "le rythme et la musique", l'observatrice accomplit

29 Dyane Léger, Les anges en transit, Moncton/Trois-Rivières, Perce-Neige/Écrits des Forges; 1992. 
la fusion totale et rédemptrice entre la survivance acadienne (cajune) et celle des Noirs: "Ces hommes sont fiers. Ces hommes sont noirs. Ils s'appellent Le Blanc's taxi, Richard's taxi, Allain's taxi, "At your service Mame!!!" Ils parlent, dansent, roulent des hanches en sifflotant: "Summertime... and the living is easy..." "30 Ici, l'euphorie est à son paroxysme, puisque la Nouvelle-Orléans/New Orleans permet de transcender la communauté d'origine, opprimée et oppressante, en déplaçant simplement l'axe de la grande dispersion. L'évocation de cette ville, aux frontières de la Louisiane cajune, permet de préserver la fiction fondatrice de l'implantation continentale de la communauté acadienne, au point où elle investit tout l'héritage culturel afro-américain et semble l'avoir précédé.

Il y a définitivement quelque chose de vampirique dans ce voyage appelé "New Orleans". Des choses qui ne se passent pas ailleurs. Dans les autres villes. Des choses qui n'ont rien à faire avec les chaises berceuses qui ne savent pas avoir d'enfants. Des choses souterraines qu'il vaut mieux ne pas montrer en plein jour. ${ }^{31}$

Culture de la sous-jacence, communiquant avec toute l'Amérique marginalisée qu'elle absorbe par des voies "souterraines", la culture acadienne parvient, chez Léger, à briser les conditions de son espace restreint.

Pour bon nombre de poètes de la renaissance acadienne, donc, la ville pauvre et opprimée que symbolisent les quartiers noirs de New York (Harlem est mentionné explicitement chez Ulysse Landry et Raymond Guy LeBlanc. par exemple) et de New Orleans (chez Gérald Leblanc et Dyane Léger, par exemple) se donne à lire dans sa transparence fulgurante: accents musicaux, rythmes et nomadismes. La ville noire est le substitut métaphorique de la mer: fugitive et nourricière. Elle assure la lisibilité même de la dispersion. Et ainsi il en va d'une grande solidarité de l'oppression, traversant les interdits de la race et de la langue. Cette conscience transversale fusionne toute l'Amérique urbaine de Moncton à New York.

Enfin, il faut le dire, le surgissement de la "cité" dans l'imaginaire poétique acadien, c'est aussi bien souvent celui de la 
32

langue anglaise. Très farouchement francophones, la plupart des poètes acadiens des vingt-cinq dernières années ressentent pourtant profondément leur allégeance à l'anglais. C'est donc aussi par sa complicité linguistique et culturelle avec New York City et New Orleans que Moncton a pu s'imposer comme un espace urbain privilégié, porteur de rédemption. Aux yeux de Herménégilde Chiasson, la présence interstitielle - parfaitement concertée - de l'anglais faisait toute l'audace et la modernité d'Acadie Rock de Guy Arsenault. La liberté, c'était celle de clamer l'anglais à la face de la grande famille acadienne normative. Si la ville semblait résolument ouverte, c'était donc parce qu'elle permettait une gestion plus souple des langues, une diglossie non plus entachée de culpabilité, mais productrice de spécificité et de modernité. Ainsi, dans l'imaginaire de l'Acadie urbaine, de Robichaud à Moncton à "New York City", l'acte d'écriture glissait insensiblement vers la langue métamorphique de l'Autre, celle de toute l'Amérique. Il n'y aurait de libération que dans cette langue. Dans Acadie Rock, Guy Arsenault n'avait pu qu'entrevoir l'immense potentiel d'ouverture intellectuelle dont Moncton serait la métonymie restreinte et New York City / New Orleans les métaphores triomphantes.

Cette construction de la cité acadienne a-t-elle pris fin au cours des dernières années? Absente des écrits plus récents d'Herménégilde Chiasson ou de Daniel Dugas, par exemple, elle ne subsisterait plus que dans l'ouvre de Gérald Leblanc, mais à l'état sublimé et nostalgique. Cela n'est pas sûr. D'abord, la ville restreinte s'est déplacée vers la métropole américaine, dont elle n'est toujours à l'heure actuelle qu'un reflet. Moncton, c'est un peu New York City, un peu New Orleans / La Nouvelle-Orléans, un peu Harlem, un peu Soweto ("Moncton Mandela "32), un peu San Francisco. En fait, la "civitas" acadienne, poussée par la "dérive / drive" (Leblanc), "dévire" (LeBlanc), n'a cessé depuis les premiers textes poétiques de s'étendre aux confins du monde, de sorte que Moncton se situe désormais dans un imaginaire à la confluence de toutes les villes, de toutes les cultures, de toutes les oppressions.

Et puis c'est chez Serge Patrice Thibodeau, contre toute attente, que resurgit encore maintenant l'imaginaire urbain. Portée par un puissant mysticisme et la quête acharnée de l'exotisme, la 
poésie de Thibodeau semble d'abord rejeter sans compromis l'Acadie natale, étroite et sans espoir. Mais le compte n'est jamais tout à fait réglé, car métaphoriquement et littéralement le poète dépaysé revient toujours se tapir dans le village de l'enfance. La quête de la connaissance ne conduit pas, chez lui, à une fracture de la communauté d'origine qui reste, elle, intacte, dans son attente du retour homérique du poète. Chez Thibodeau, la "civitas" acadienne est plutôt entièrement déplacée. Elle ne saurait se construire, cette fois, dans l'Acadie restreinte: Moncton, Caraquet ne suffisent plus. C'est d'ailleurs l'absence de métropoles habitables qui, pour Thibodeau, condamne irrémédiablement l'Acadie. Du Cycle de Prague surtout au Quatuor de l'errance, l'avenir se résume plutôt à la succession des villes traversées par l'errant, lieux liminaires où transite le poète, animé par une ouverture culturelle absolue. Prague, New Delhi, Téhéran, Karachi, Jérusalem, Mexico, Montréal (dans Nocturnes, 1997), entre autres, la litanie suffit à elle seule à susciter le dépaysement. De toutes ces villesseuils, c'est sans doute New Delhi qui, au départ, semble la plus chargée de sens. Ville "nouvelle" encore ici, comme New York City et New Orleans, New Delhi offre, au début du Quatuor de l'errance, l'image de la rédemption tant attendue. Cette rédemption tient à l'effort de négativité qui anime le poète errant à son entrée dans la cité indienne. Incapable de cet acte de négativité dans l'espace natal, il se sent alors en mesure de rejeter les conditions d'enfermement qu'il associe à la communauté acadienne:

Aux lisières des bidonvilles de l'espoir, mes yeux s'illuminent.

Autrefois, l'affront fut un gîte, la colère, une table.

Préférer aujourd'hui le ciel bleu de Delhi, ses rues, ses cris. ${ }^{33}$

Cité de l'irréel, de la transformation de la matière, de l'appauvrissement stratégique: telle avait été New Delhi. Chez Thibodeau, la ville légendaire, où se produit à tout coup et dans la perte de soi la renaissance identitaire, n'en est pas moins le lieu d'une profonde inadéquation aux choses et aux êtres. C'est son exigeante irréalité, sa pauvreté matérielle. C'est pourquoi, dans le Quatuor de l'errance, le tracé de la ville aboutit inévitablement à Jérusalem où le poète, condamné à mort, doit faire son entrée pavoisée. "Jérusalem est venue dans ma vie me hanter." 34 Tout

33 Serge Patrice Thibodeau, Le quatuor de l'errance, suivi de La traversée du désent, Montréal, l'Hexagone, 1995, p. 20.

34 Ibid., p. 220. 
34

semblait converger vers ce moment, faisant du Quatuor de l'errance le point culminant de l'invention sacralisée de la ville. New York, New Orleans, Prague, New Delhi, Amman, toutes ces villes n'avaient pu être que les étapes d'une lente cérive vers la cité apocalyptique, la Jérusalem céleste. Plus rien ici ne reste des lieux familiers qui, comme chez Gérald Leblanc, donnaient un semblant de matérialité à l'espace urbain. Dans l'œutivre de Thibodeau, la succession incantatoires des villes étrangères s'ouvre sur une idéalisation de la pureté des pauvres, un mysticisme de la négativité qu'Alain Masson avait déjà bien repéré vingt-cinq ans plus tôt.

Ainsi, faut-il le dire, la "civitas" acadienne - à l'exception peut-être de l'œuvre de Serge Patrice Thibodeau - n'aura pas été tout à fait l'émergence en poésie d'une conception postmoderne de l'espace urbain. Rejet de la communauté uniforme et dirigiste, brouillage des valeurs traditionnelles, pour reprendre autrement les termes de Gilles Lipovetsky: la ville de Leblanc, Chiasson, Léger, Landry ou Thibodeau a été,certes, tout cela. Mais la poésie de la renaissance acadienne ne s'est pas portée uniquement à la défense de l'individualisme. Peut-être aurait-elle souhaité, par ses premiers "cris", mettre fin une fois pour toutes à la "prééminence de la centralité "35, mais, dans l'angoisse de la marge culturelle, comment donc risquer de tout perdre? Par la ville inventée, cette "Acadie City" des poètes, il fallait préserver la "légende". Or la "légende" n'a pu avoir de "lisibilité" que dans la reconnaissance d'une oppression, archéologique, continentale, planétaire (chez Serge Patrice Thibodeau) où s'inscrit l'histoire de l'Acadie. De la ville sauvage de Raymond Guy LeBlanc à la Jérusalem céleste de Serge Patrice Thibodeau, voilà que la "cité" s'abolit malgré tout dans des formes insoupçonnées de proximité et de solidarité. Le poète fait toujours son entrée parmi les siens. En apparence effacement de la communauté, lieu de son essouflement final, la "cité" rêvée n'aura été, dans le discours poétique du moins, qu'une autre manière d'envisager, sur un plan utopique ou même mystique, le destin collectif.

35 Gilles Lipovetsky, L'ère du vide. Essais sur l'individualisme contemporain, Paris, Gallimard, [1983], 1993, p. 17. 